\title{
TOWARDS INDUSTRY 4.0 IN CORPORATE ENERGY MANAGEMENT
}

\author{
HORST JUNKER ${ }^{1} \&$ CARSTEN DOMANN ${ }^{2}$ \\ ${ }^{1} \mathrm{IMBC}$ GmbH, Germany \\ ${ }^{2}$ bbw Hochschule, Germany
}

\begin{abstract}
Industry 4.0 is the "networking of autonomous, situationally self-controlling, knowledge based, sensor supported and, spatially distributed production resources incl. their planning and control systems" (Arbeitskreis Industrie 4.0). With ISO 50.001 an industry norm is available, improving the corporate energy efficiency through its implementation. For that ISO 50.001 demands to formulate a company-wide energy policy, to ensure the determination of the organization and information structures, and is designed for the target of energy efficiency improvement. Overall, the topic addressed by ISO 50.001 is so complex that IT support is required. Actually, more than 120 software systems are offered on the market to support corporate energy management. When checking these systems their very coarse-granular character becomes obvious. A detailed system, regarding single industrial facilities' collection of energy consumption data, is not possible, with a result that exclusively involves a monitoring and reporting function. Planning and controlling is not supported. Thus, existing software systems have elementary deficits concerning Industry 4.0, because they are neither able to receive data (sensor supported) in real time, nor do they have (automated) planning and controlling components based on respective knowledge-based systems, business intelligence and big data procedures. We developed an energy management system with the possibility to collect energy consumption data finely granular on the level of production facilities based on data sheets of plants manufacturers. Further, a modular and self-organizational communication system (as a hard/software combination) has been developed that "reads" internal electrical energy data from the production facilities in real time. A further system enhancement consists of sensor-supported flow measurement of fluid or gaseous energy sources and real-time provision for the energy management system. In this way, an essential component of an Industry 4.0 system for the corporate energy management is realized: the detailed and timely provision of energy consumption data. A last step for developing a corporate energy management system conforming the Industry 4.0 requirements procedures off descriptive data analysis, optimization algorithm and simulation processes shall be developed and big data analyses and business intelligence procedures shall be applied. These optimization procedures shall achieve Industry 4.0 criteria of selfdependent planning and controlling.

Keywords: Industry 4.0, ISO 50.001, Energy Informatics, operational energy management, operational efficiency enhancement, Big-Data-Applications, power consumption data, SME.
\end{abstract}

\section{INTRODUCTION}

As shown in the following sections, for some time the scientific community agrees on the fact that operational energy management is a relevant and profitable application area for procedures and technologies of Industry 4.0. Apart from that the operational management in general is submitted to the practical realization of ISO-Norm 50.001. According to the small number of interfaces between procedures and technologies of Industry 4.0 and the procedural rules of ISO 50.001 it seems to be basically possible to realize IT-supported energy management systems with reference to ISO 50.001 as well as Industry 4.0 to achieve a greater degree of energy efficiency. Despite the expectable efficiency enhancements, there haven't been published any IT systems in practice that merge all requirements of ISO 50.001 and Industry 4.0. 
To realize such systems the existing IT-supported operational energy management systems shall be complemented vertically in two directions. At first operational energy related consumption data must be collected on a timely manner, if possible. The necessary main/software combinations have been already realized, as it will be shown below. Secondly the collected data must be subordinated to the optimization algorithms to achieve the impulse of IT-supported procedures. The latter shall optimize the provision and the consumption of all operational amounts of energy (as well as) with the meaning of cost efficiency.

Until the operational energy management systems according to ISO 50.001 have been complemented by those two components it can be managed to harmonize ISO 50.001 and Industry 4.0 effectively and to optimize the operational energy efficiency. Therefore, it will be described below how these optimizing algorithms shall be developed.

\section{FUNDAMENTAL ASPECTS OF INDUSTRY 4.0}

The task force Industry 4.0 subsumes in its "Implementation recommendations for the future project Industry v4.0", "a linkage of autonomous, situational self-managed, self-configured, knowledge based, sensor based and spatially distributed production resources (production equipment, robots, conveyor- and storage systems, and equipment) inclusive its planningand controlling system". Ultimately, all relevant aggregates for the production shall

- $\quad$ provide (sensor-supported) real time data relevant for planning and control and

- optimize operational manufacturing through the application of knowledgebase systems and procedures.

The inclusion of holistic determinant factors for manufacturing and due to its complexity an extremely high entitlement has been formulated that cannot be easily redeemed in practice, it is only possible under considerable difficulties and with high effort related thereto.

The above-mentioned complexity as well as the inner condition of Industry 4.0-systems requires mandatory IT support. Such systems focused on Industry 4.0 Technologies that are oriented on the energy management of a company haven't been discussed - except several general indications highlighting the possibility to use such Industry 4.0 for the operational sphere - neither in practice nor in scientific literature.

The term Industry 4.0 was created in 2011 and has been used inflationary for different areas of application and the development of technologies and for this reason there is a lack of clarity and uncertainty in other areas (not only terminological). Following this fact and due to the short time frame, it has not yet succeeded by the science to (finally) interfuse the complex area of application of Industry 4.0.

A significant and fundamental hallmark of Industry 4.0 consists in the use of possibilities, arising from networking of technical systems in real time.

Cyber-physical systems qualified in recording and processing decentralized and in real time data of machines and assets, with available sensor technology, are the basis of Industry 4.0 systems.

On this (technological) basis to capture a digital shadow, a high number of data from different resources has to be structurally merged. Difficulties need to be resolved resulting from date resources with different transmission rates, different sensor principles and different time requirements, those shall be real-time-capably integrated. The digital shadow indicates sufficiently the image of operational reality. It is necessary to describe all required data formats, data choice and necessary levels of granularity.

Through the provision of data of the digital shadow it is possible to assess past oriented data with the use of procedures of descriptive data analysis. Forecasting models can be elaborated when using models and methods of predictive analytics. Finally across the use of 
optimization algorithms and simulation procedures there can be generated specific operating procedures. Assessment tools are focused on Big-Data-Procedures, that are convenient for processing varied data types and data structures from different data resources. However, Big-Data analyses differ from nearby traditional Business-Intelligent-Applications not only through the acceptance of different, and also unstructured data, but especially on what questions of potential methods (can be) worked on. Business Intelligence aims to measure the performance of a company (or part of it) towards the past through the use of a set of metrics. Big-Data-Applications are focused to detect patterns, their identification and forecast as well as their derivation of operating procedures, provided the digital shadow of operational reality has been projected as precisely as possible. Algorithms from the area of machine learning and artificial intelligence (AI) can help to create better results of Big-Data-Procedure.

Concerning the formerly described frame of Industry 4-0-Systems exists - at least on this abstract level - a high level of consensus in science and practice. However, it has to be acknowledged that there are several open technological questions for the creation of the digital shadow. Especially the set of method that shall be activated in the area of Big-DataApplications has not yet been investigated and developed comprehensively.

\section{INDUSTRY 4.0 FOR THE OPERATIONAL ENERGY MANAGEMENT}

"Industry 4.0 for the operational Energy Management" can be - from a scientific perspective - assigned to the scientific area of Energy Informatics. The relevant scientific journals addressed Energy Informatics in three magazines.

In 2013, Appelrath et al. [1] presented Energy Informatics submitted in the journal's editorial Informatik Spektrum that eight of the publications will "display the wide range of topics on Energy Informatics". A detailed review of these publications elucidated, however, that the presented Energy Informatics does not at all deal with phenomenon, resulting in IT support at operational Energy Management.

The journal Wirtschaftsinformatik (Goebel et al. [2]) addressed this discipline in its principle essay, especially under the aspect of intelligent energy-saving-systems. Whereas the Energy Informatics mainly discusses problems in the fields of energy production, especially energy allocation, the intelligent energy-saving-concepts on the other hand are focused on operational fields of applications. And thus, fields of applications mentioned, can also be addressed by Industry 4.0.

The following scientific challenges of Energy Informatics regarding intelligent energysaving-systems are (Goebel et al. [2]):

- "On the first level (...) ICT enables individuals and companies to better measure their power consumption, to understand it and to react accordingly". ICT-Systems that are able to monitor and to report will be demanded. As a result, a range of market services will be addressed that are covered by current available IT-supported energy management systems, but on a (mostly less) detailed level.

- "On the second level ICT enables each person and companies to better control their power consumption through appropriate actuators", even though such actuators do not need to be IT systems. An Energy Manager or agent can also be addressed. From the perspective of Goebel et al. [2], only systems like premises and housing technology comprising areas of applications like heating, climate and lighting.

- "On the third level the power control circuit will be embedded through innovative ICT-Systems that are able to automatically tune the power consumption with the real demand (...)". It depends on systems that need to be invented recognizing and 
forecasting the real final consumption and to control the service provision to minimize the power consumption. With this perspective, a service spectrum of ICTSolutions will be addressed that is already covered by the requirements of Industry 4.0.

In the context of challenges for the science the authors list five scientific questions to concretize the Energy Informatics "in connection to the Energy Saving-Systems". Only one of these scientific questions touches the presentation of problems in connection to Industry 4.0 on operational Energy Management: "How can a large number of sensor data preferably optimal stored and reproached from an applications perspective for visualization as well as control?"

Essential aspects arising from the Industry 4.0 definition, especially planning and optimization cannot be seen as relevant research task.

Another important input was provided by Staake and Appelrath [3] in the journal Informatik Spektrum editorial focused on Energy Informatic and provided five articles in total. They give the impression that these articles represent "54 scientific and practical contributions" of Energy Informatics. None of these articles exemplified any issue of IT support of the operational Energy Management.

This results in a serious gap in the application oriented research for planning, control and optimization of operational IT-supported Energy Management Systems. These gaps need to be filled in, because and due to the initiated energy policy the operational costs for power consumption will further increase that have an impact on companies to provide contributions for the reduction of greenhouse gases.

Industry 4.0 and Energy Management have a common aim of operational efficiency enhancement. Therefore philosophy, procedures, techniques and tools of Industry 4.0 and Energy Management shall be integrated to the "Operational Energy Management Industry 4.0-System".

\section{IT SUPPORT OF OPERATIONAL ENERGY MANAGEMENT CURRENTLY USED IN PRACTICE}

Beyond the claim (of the German Government) to realize Industry 4.0 - considering the reduction of greenhouse gas emissions - an increase of operational energy efficiency is demanded. It is suggested to integrate energy management systems into the operational business. In 2012 the industrial standard ISO 50.001 was installed and since then it is intended to increase companies' energy efficiency systematically and continuously. It is rightly - assumed that the standard can be modified according to company specific requirements.

Basically ISO 50.001 follows the ISO-Management systems (e.g. quality, environment, sustainability) in order to ensure a constant implementation of "Plan-Do-Check-Act" circles for a continuous improvement process. Companies applying ISO 50.001 are obliged to collect all of their energy streams systematically that provides the opportunity - in case of upcoming investment decisions - to consider energy efficiency aspects comprehensively.

An essential task in this application is to draft a companywide energy policy (repeatedly) and to secure its compliance through the application of further ISO 50.001 tasks. All organizational and informational structures to be integrated should be recorded and shall be created with the objective of increasing the energy efficiency. The success of these methods shall be internally audited on a regular basis caused in elaboration of a differentiated energy policy by the companies' management. 
Once again, an essential hallmark of ISO 50.001 consists on the ability to adjust (all) company specific requirements. ISO 50.001 (as all ISO-Management Systems) is to be understood as a guideline with the possibility of several expressions. The short draft of the standard clearly revels that

- the formulation of energy policy

- planning

- its implementation and operation

- measurement control

- monitoring and adjustments

within the energy management comprise such volume of activity that is suitable to claim IT support. This claim has been recently taken into account. It was investigated (Junker et al. [4]), that since the beginning of last year approximately 120 standard software systems have been available to support the operational energy management. A critical review of these systems indicated that more than one-half of them meet the needs through ISO 50.001. The software systems typically are characterized to be universal and due to this fact able to catch and to cover all different operational individualities.

The critical review of the software systems for operational energy management offered on the market shows furthermore that these systems are construed unanimously granular to redeem the postulated universal validity. They actually take into account that the finally structured - for instance with reference on specific production facilities - power (consumption) data do not exist. With regard to the electrical current as source of energy only the consumption data of annual invoices of energy suppliers is available. The same also applies to other energy carriers. With that in mind it becomes clearly that software systems consist of a monitoring and reporting element to (nearly) increase the operational power efficiency. Other tasks like planning and controlling of an (ambitious) energy management won't be software based supported. At best these software systems are able to provide vague indications for planning and controlling that requires an intensive management interpretation.

Altogether and consequently postulated all currently available software systems on the market for the energy management with regard to Industry 4.0 requirements contain fundamental deficits. On the one hand, no (automated) planning and control components (based on knowledge-based systems, business intelligence methods or Big-Data procedures) do exist. On the other hand, there is no intending that these systems have a provision of energy or energy-related data (sensor controlled) in real time, even though it must be available as essential databases for automated planning and control components.

\section{IT-SUPPORT TRENDS FOR OPERATIONAL ENERGY MANAGEMENT SYSTEMS}

IMBC GmbH developed a software system in the past to support the operational energy management; its functionality mainly is located in the same mainstream as other existing software systems. Here the attempt has been to collect the fine-grained power consumption data on the level of production systems.

In order to achieve this (first approximation) they wrote off their initial claim on general validity in favor of industry-oriented solutions. So far there are two solutions: One solution for the printing industry and one solution for the metalworking industry. Within two sector-specific company networks each typical production system as well as production machines have been investigated and their energy related parameters have been tracked into single data bases. The energy parameters have been collected from the assets' and machine manufacturers' data sheets or have been allocated from the manufacturers. The respective power consumption is determined by multiplication with the operating hours. Although a 
software system exists that can provide more detailed information about operational energy efficiency compared to most IT supported energy management systems, but randomly tested measures on different user companies have shown that real life power consumption significantly differ from the provided manufacturers' data sheets. Especially the real time power consumption within a time schedule is subject to fluctuations (e.g. peaks after the system started). The data sheets of the production facilities and production machines collect a variety of operational data (mostly service purposes) including energy related data, with the limitation however of electrical power as source of energy.

Based on this knowledge IMBC $\mathrm{GmbH}$ developed a modular and self organized communication system that can be used for existing energy management and monitoring systems and furthermore supplements them. A modular communication module was developed that can be flexibly customized to the provided requirements of the asset's entry and exit. Former systems are designed only for a special monitoring or communication system (WLAN, LAN, Bluetooth, etc.) only possible to a limited extent.

For this reason both interface standards - monitoring system as well as measurement object - have been designed and developed. In order to guarantee the interface's function flexible and independently to consider all the demands which could arise in practice a special library has been developed, by implementing all these relevant parameters. This library enables a smooth communication between measuring points on the machine and the communication module. The library can interact with common machine toll standards. The collected data will be aggregated in a specially developed data format. It analyzes only relevant data (just consumption data or control data) for the monitoring systems.

Physical Signal recording takes place with special plug in module that can be added to the communication module based on the modular system design and hardware requirements. The data output transmitted to the monitoring system takes place through a special independent interface.

A special feature of this system is its ability to communicate self-organized. Through the connection of the communication modules the whole system can react flexible on external impacts as they influence the system's communication and its measurement capability. This means for instance that the system automatically can evade to an alternative communication channel in case of a WLAN interruption caused by the metal body of a forklift truck. A secured and exact data collection shall be guaranteed on every time even under adverse and constantly changing transfer conditions.

There has been created as system having the following functionalities:

- modular structure of the hardware system: use of standard hardware (e.g. processor, communication tools for Wi-Fi/Bluetooth, etc.) to keep the hardware price as low as possible;

- low price for communication module to increase consumer acceptance of SME

- Support of different communication types (LAN, WLAN, Bluetooth, BUS-Systems, etc.) for the data collection between asset/machine and the central energy monitoring cockpit; further protocols/communication systems can be updated with plug-ins;

- generic interface to get access to the measuring instruments' data (use of different communication protocols collected in a library) to waive several proprietary manufacturer-specific systems in favor of freely selectable evaluation software

- Aggregation of separate information in a specific data container/format for data transfer; 
- system's ability of self organization through the firmware of the communication module;

- flexible and customized system's expandability through support of different communication tools and the system ability of self organization considering a new purchase renders unnecessary;

- technical basis for the entire company without media discontinuity energy monitoring (focused on SME use).

According to estimations from experts, hardware costs for the serial production per module will be below 50.00 Euro.

According to the above-mentioned development of a hard/software combination to collect electrical energy data in a timely manner from machines and assets and to transfer the data to another server a current project under development shall solve the deficits in real time collection for the "through-feed measurement" of liquid and gaseous energy carriers caused by existing IT-supported energy management systems. The objective is to develop a modular and self-organized communication system updating the existing IT support of existing energy management and monitoring systems.

In the first step market available and frequently used monitoring software systems will be technical analyzed according to their requirements on technical data input. At the same time there will be done an investigation of output options of the measuring instruments for the "through-feed measurement" of liquid and gaseous energy carriers. This will be used to render which formats should support the planned development. A conception and development of both interface standards will follow (focused on monitoring system and measurement object). With respect to the interface the same requirements shall be met as realized in the former project. This means the physical signal collection takes place through special plug in module that can be added to the communication module due to its modular design and according to the hardware requirements. The data output to the monitoring system takes place through a special interface working independently to the used monitoring software. The ability of self-organization of communication will be ensured.

The manufacturing of the final prototype, which means the boards' layout-design, the choice of components as well as the fitting of circuit boards can be realized through third parties. Preliminary considerations on hardware costs (provided that serial manufacturing will take place) result in costs that do not exceed an amount of 50.00 Euro for each hardware module.

Considering the results of the existing system and the system under development errors in energy monitoring of currently available energy management systems at SME can be overcome. Especially real time energy data and times, short as pleasure can be recorded and collected.

\section{DEMAND ON SCIENCE AND DEVELOPMENT FOR THE REALIZATION OF INDUSTRY 4.0 WITHIN OPERATIONAL ENERGY MANAGEMENT}

The current status of IT support of operational energy management has to be compared with the formerly drafted characteristics of Industry 4.0 applications to derive a scientific goal for upcoming projects.

Junker et al. [4] have found that since the beginning of last year approximately 120 standard software systems have been available to support the operational energy management. A critical review of these systems indicated that more than one-half of them meet the needs through ISO 50.001. ISO 50.001 (as all ISO-Management Systems) is to be understood as a guideline with the possibility of continuous several expressions. The 
developed task spectrum (as additional decisive feature of this standard) is characterized to be universal and due to this fact able to catch and to cover all different operational individualities.

Energy Management Systems have been constructed unanimously granular (obviously to redeem the postulated universal validity). They actually take into account that the finally structured - for instance with reference on specific production facilities - power (consumption) data do not exist. With regard to the electrical current as source of energy only the consumption data of annual energy supplier invoices is available. The same also applies to other energy carriers. With that in mind it becomes clearly that software systems consist of a monitoring and reporting element to (nearly) increase the operational power efficiency. Other tasks like planning and controlling of an (ambitious) energy management won't be software based supported.

Currently existing IT systems require additional functionalities to increase the operational energy efficiency as they result in the requirements that have to be met by Industry 4.0 Systems. On the one hand procedures need to be developed to redeem the requirements on real time processing of Industry 4.0 Systems. On the other hand processes, methods and technologies should be developed which could help to necessarily intervene in the operational energy management system. Finally, the overall energy efficiency of the companies' production sphere can be optimized. Especially the development of such optimization approaches - and its integration into existing software systems to increase the operational energy efficiency - represents the scientific target of this project idea.

\section{REFERENCES}

[1] Appelrath, H.-J., Mayer, C. \& Steffens, U., Editorial. Informatik-Spektrum, 1/2013.

[2] Goebel, C., et al., Energieinformatik - Aktuelle und zukünftige Forschungsschwerpunkte. Wirtschaftsinformatik, pp. 31-39, 1/2014.

[3] Staake, T., Appelrath, H.-J., Editorial. Energieinformatik, 2/2015.

[4] Junker, H., Meyer, A. \& Sangmeister, J., Handbuch Standardsoftware im Betrieblichen Umweltschutz - Auswahl, Anwendung und Produkte, Erich Schmidt Verlag: Berlin, 2015. 